\title{
Prevalence, age of onset and demographic relationships of different areca nut habits amongst children in Tower Hamlets, London
}

\author{
P. Farrand, ' R. M. Rowe, ${ }^{2}$ A. Johnston, ${ }^{3}$ and H. Murdoch, ${ }^{4}$
}

Objective To examine prevalence and demographic relationships of different areca nut habits amongst children.

Design Self-administered questionnaire.

Subjects Children aged between 11 and 15. Of 800 questionnaires distributed, 704 were fully completed (88\%). Setting Two secondary schools in the London district of Tower Hamlets.

Measures Demographic, areca nut habits used, age first used, still using, frequency of use.

Results Users of any areca nut habit were exclusively from the South Asian population. Of this population, $77 \%$ had engaged in a habit, and dependent upon habit between 54 and $92 \%$ of these still remained current users. The highest prevalence of current use for boys and girls respectively was for areca nut alone $36 \%$, $43 \%)$, followed by mistee pan $(35 \%, 29 \%)$, betel-quid $(27 \%$, $26 \%)$ and pan masala $(14 \%, 16 \%)$. Of the current users, $44 \%$ engaged in one habit only, $24 \%$ two, $20 \%$ three and $13 \%$ all four. The highest period of risk for starting to use areca nut alone, betel-quid and mistee pan was between ages 5 and 12, whilst for pan masala it was after 10. Boys had a significantly higher risk of beginning use before $10(P<.001)$ and a higher frequency of use for pan masala $(P<.01)$, areca nut alone $(P<.05)$ and betel-quid $(P=.06)$ than girls. The frequency of using each habit was between 3 and 5 episodes per week, however boys use pan masala approximately 10 times per week.

Conclusion South Asian children may already be experienced users of areca nut. Greater attention should be directed towards identifying signs of oral submucous fibrosis, oral cancer and other potentially malignant lesions within the South Asian population.

$\mathrm{T}$ he areca nut habit has a major social and cultural role in communities throughout the Indian subcontinent, South-East Asia and parts of the Western Pacific. Following migration from these countries into the UK, predominantly into inner city areas, the habit has remained prevalent. Estimates of the habit vary

${ }^{1}$ Lecturer in Medical Psychology, Department of Human Science and Medical Ethics, St Bartholomew's and the Royal London School of Medicine and Dentistry, Turner Street, London E1 2AD; ${ }^{2}$ Research Worker, MRC Child Psychiatry Unit

and Centre for Social Genetic and Developmental Psychiatry, Institute of

Psychiatry, De Crespigny Park, Camberwell, London SE5 8AF; ${ }^{3,4}$ Medical

Student, St Bartholomew's and the Royal London School of Medicine and

Dentistry, Turner Street, London E1 2AD

${ }^{*}$ Correspondence to: Dr P. Farrand

email:P.A.FFARRAND@MDS.QMW.AC.UK

REFEREED PAPER

Received 8.03.00; Accepted 19.07.00

(c) British Dental Journal 2001; 189: 150-154 between $66 \%{ }^{1}$ and $95 \%{ }^{2}$ within the Bangladeshi UK communities, but less within the UK's Indian (15\%) and Pakistani communities $(7 \%) .{ }^{1}$ The emphasis in these studies has been exclusively upon the betel-quid (paan) habit in adults. Percentage of users ${ }^{3}$ and frequency of use increases with age and retrospective report indicates the betel-quid habit predominantly begins between age 11 and 15, although a minority started before age $5 .{ }^{4}$ However other areca nut habits exist ${ }^{5}$ and differences between these have not been extensively examined (Table 1).

Within the Bangladeshi community, $92 \%$ of females and $39 \%$ of males add tobacco to their betel-quid. ${ }^{6}$ This difference is explained by religious and social restrictions placed upon females smoking cigarettes.

Countries with a high prevalence of the areca nut habit have higher rates of oral cancers. ${ }^{7}$ However it is the addition of tobacco, rather than simply the habit itself, which may be associated with such rates. ${ }^{6,8-10}$ Associations between areca nut without tobacco and oral lesions such as oral submucous fibrosis (OSF) and leukoplakia are well-established however. ${ }^{6}$ A relationship between the risk of developing OSF and habit has also been documented. ${ }^{9}$ In a case controlled study carried out in Pakistan comparing areca nut alone, betel-quid and betel-quid with tobacco the highest relative risk (RR) of developing OSF was amongst chewers of areca nut alone $(\mathrm{RR}=154)$. This was significantly higher than the relative risk posed by betel-quid with or without tobacco $(R R=64$ and $R R=32$, respectively). ${ }^{11}$ Another case controlled study from India reported

Table I Different areca nut habits

\begin{tabular}{|c|c|}
\hline Habit & Notes \\
\hline Betel-Quid & $\begin{array}{l}\text { A mixture of sliced areca nut and slaked lime (from } \\
\text { limestone or coral) and also often tobacco is placed within a } \\
\text { betel-leaf. The leaf is folded, and stored in the buccal } \\
\text { mucosa and gently chewed or sucked. Other substances } \\
\text { are added as per local preferences. Also commonly } \\
\text { referred to as paan, pan or paan-quid. }\end{array}$ \\
\hline Pan Masala & $\begin{array}{l}\text { A concentrated powdered proprietary form of the above. } \\
\text { Other substances, including tobacco are added as per local } \\
\text { preferences. Often commercially sold as pan parag. }\end{array}$ \\
\hline Areca Nut Alone & $\begin{array}{l}\text { Areca nut is sliced and chewed on its own. When packaged, } \\
\text { sweeteners are added and sold as sweet supari, gua or } \\
\text { mawa. }\end{array}$ \\
\hline Mistee Pan & $\begin{array}{l}\text { Commercially prepared boiled 'sweets' containing areca } \\
\text { nut and various sweeteners. Prepared and packaged to } \\
\text { make it particularly suited to younger children. Also known } \\
\text { as mitha pan. A form known as gutka that has tobacco } \\
\text { added has recently become available. }\end{array}$ \\
\hline
\end{tabular}




\section{community dentistry}

all areca nut habits to have a relative risk of 109.6, whilst the combined use of areca nut alone and betel-quid had a relative risk of 780.0. ${ }^{12}$ A dose-response relationship between degree of exposure and the risk of developing OSF has been proposed. Whilst a high frequency of use has been reported to increase risk, ${ }^{11,12}$ duration of use has also been implicated. ${ }^{12}$

Given that the relative risk of developing OSF varies tremendously by areca nut habit, it is important to establish the prevalence and frequency of use across the various habits. This study extends previous research ${ }^{1-4,13}$ by widening the focus from betel-quid to the other areca nut habits. Additionally as the focus of this study is upon children aged between 11 and 15 two further issues can be addressed. First, the extent to which the areca nut habit is being continued within the South Asian population amongst children who are predominantly first or second generation UK born. Second, the extent to which the habit is extending amongst children with other ethnic backgrounds.

\section{Methods}

\section{Subjects}

143 boys $(20 \%)$ and 561 girls (80\%) aged between 11 and 15 attending two comprehensive secondary schools within the Tower Hamlets Local Education Authority (LEA), rated as the second most deprived LEA in the country. Tower Hamlets, an inner-city borough of East London with the largest Bangladeshi population in the UK, has high levels of social and economic deprivation. Four schools in Tower Hamlets were initially contacted to assist in the study, of which two were willing to participate as the others were currently engaged in a non-related study. One of the schools was mixed sex, consisting of 450 pupils aged between 11 and 15 years of which $70 \%$ participated in the study. The other school was all girls with 832 pupils aged between 11 and 15 of which $52 \%$ participated in the study. Both schools were highly representative of the others in the area, with the general school intake of Tower Hamlets reflecting the low socio-economic characteristics of the population. Government school OFSTED reports indicate that between 70 and $84.2 \%$ of pupils attending schools in Tower Hamlets are entitled to free school meals compared to national figures of between 12 and $22 \%$. Of the schools taking part in the study, 78 and $84.2 \%$ of the pupils were eligible for free school meals.

Self-reported ethnic background of the pupils was predominantly 'Bangladeshi' (498, 70\%), along with 'Asian' (92, 13\%), 'British' (82, 12\%), 'Pakistani' (16, 2\%), and others (16, 2\%). Eight hundred questionnaires were delivered to the participating schools and distributed within randomly selected class registration sessions. Seven hundred and four questionnaires were fully completed (88\%) and $52(7 \%)$ were left entirely blank, possibly never having been distributed. A further $44(6 \%)$ were returned with demographic information completed but the areca nut questions left blank. This group of non-responders was composed of only $2 \%$ of the total sample who reported a South Asian ethnic origin, being made up of mostly British (20\% of the total British sample) and other ( $30 \%$ of the total other sample) children.

\section{Research instrument}

A single sided self-administered questionnaire which could be completed in less than 10 minutes was constructed using simple terminology. The questionnaire elicited demographic details (age, sex, self-reported ethnic origin), and questions concerning areca nut habits (ever used, age of first use, current use, frequency of use). A brief description of each habit was included. Additionally for pan masala and mistee pan, which are available packaged, major brand names common in Tower Hamlets were also supplied. Participants were given instructions including information on their ethical rights regarding participation before completing the questionnaire.

\section{Statistical Analysis}

Participants provide responses to each of the questions asked (ever used, age of first use, current use, frequency of use) with regard to each of the four areca nut habits. Each question was initially examined using multivariate regression models fitted to the sets of four responses including a categorical variable coding for areca nut habit. Differences in age and sex associations between habits were examined with the inclusion of an interaction between demographic and areca nut habit variable. Where such interactions were significant, analyses of each habit separately were conducted. Survey regression models that account for the clustering of multiple responses from the same individual were employed for the multivariate modelling.

Binary outcome variables were analysed using logistic regression. Count outcome variables have been analysed using ordinary regression with robust test statistics based on the heteroscedastic consistent parameter covariance matrix. ${ }^{14}$ Age at first use was modelled using the Kaplan-Meier ${ }^{15}$ survivor function $S(t)$; the probability of not having used a particular habit to time $t$ or beyond. The failure function, calculated as $1-\mathrm{S}(\mathrm{t})$, is graphed for each habit for boys and girls. Current age was measured as age at last birthday and treated as a continuous variable. Interactions between sex and age were not assessed caused by a low number of boys at each age.

\section{Results}

\section{Prevalence}

Any areca nut habit was engaged in within the Bangladeshi, Asian and Pakistani populations only. Therefore those reporting a British or other ethnic origin have been excluded from subsequent analyses.

Seventy-seven per cent (95\% Confidence Interval (CI): 74\%, $81 \%$ ) of the sample reported having used at least one habit. There was no difference in the prevalence of having used any of the habits between the ethnic populations studied $\left(\chi^{2}=.72, \mathrm{df}=2, P=.7\right)$ nor between the girls attending different schools $(\mathrm{z}=-1.29, P=.2)$.

The percentage of boys and girls that have ever used an areca nut habit are shown in Figure 1. It can be seen that areca nut alone is the most commonly used habit, with over $50 \%$ of boys and $70 \%$ of girls reporting use at some time. Pan masala is least commonly used, with less than $30 \%$ of the sample having used it any time. Approximately $40 \%$ of the sample have used betel-quid and mistee pan at some time. A multivariate model confirms significant differences in the prevalence of having ever used the different habits $(\mathrm{F}(3,602)=105.19, P<.001)$. All pair-wise differences between the prevalence of having used the different habits are highly significant $(P S<.001)$, except for the comparison between betel-quid and mistee pan $(P=.053)$.

Sex differences in ever-use of the different habits appear confined to areca nut alone where usage is more prevalent in girls. This specificity is supported by a highly significant interaction between sex and habit $(\mathrm{F}(3,602)=4.28, P=.005)$. Analyses of the effect of sex on habit individually revealed that the prevalence of ever-used areca nut alone was higher in girls than boys (Odds Ratio (OR): 2.32 95\% CI: $1.47,3.66 ; \mathrm{z}=3.61, P<.001)$ but that there were no sex differences in having used betel-quid, pan masala or mistee pan $(P S>.3)$. Age did not relate to prevalence of habit overall $(P=.3)$ and did not interact with any individual habit $(P=.3)$

Figure 1 also shows the percentage of current users. It can be seen that a substantial percentage who have ever engaged in an areca nut habit remain current users. Areca nut alone is the most common habit engaged in, followed by mistee pan, betel-quid and pan masala, this trend being the same for boys and girls. Of those currently engaged in an areca nut habit, $44 \%$ use one habit, $24 \%$ use two, $20 \%$ use three and $13 \%$ use all four habits. In these current users, there was no relationship between age or sex and the number of habits engaged in $(P S>.7)$. 
Fig. I Percentage (and 95\% confidence intervals) of children who have ever used and currently use each habit

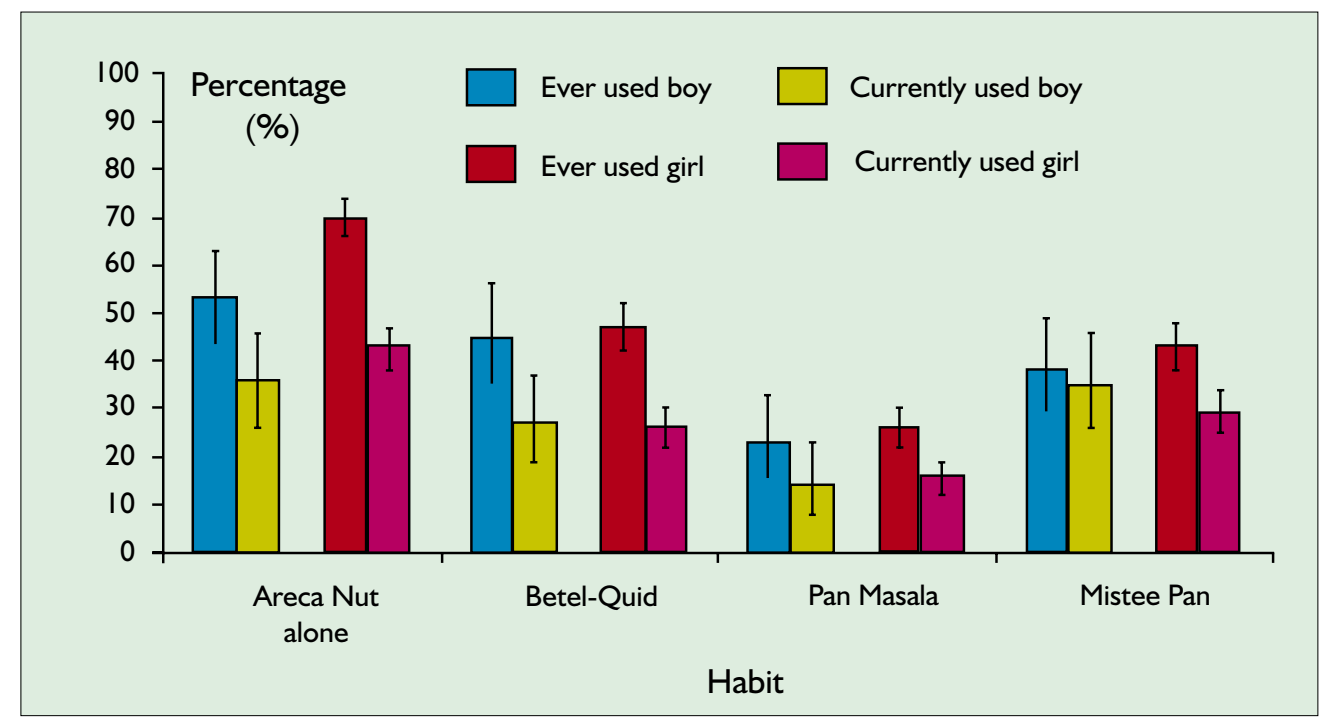

\section{Age at first use}

From the retrospective report of age at first use, the probability of having started to use each habit from birth to age 15 is shown for boys and girls in Figure 2.

Very few children started to use any habit before age 5. For boys, the highest period of risk (as revealed by the steeper curves on Figure 2) for engaging in the habit of areca nut alone, betelquid and mistee pan is between the ages of 5 and 10, with the curves then becoming flatter. Rates of pan masala uptake for boys rose steadily from 5 to 12 , with the curve becoming flat after 12 . In girls, the rate of uptake for areca nut alone, betel-quid and mistee pan remains at a largely constant rate between 5 and 12 . As with the boys, the highest period of risk for starting to use pan masala was slightly later, after 10 .

The percentage of boys and girls that started a habit before age 10 are shown in Figure 3. Differences in these percentages between the habits were highly significant in a multivariate model accounting for the effect of current age $(\mathrm{F}(3,451)=22.92, P<.001)$.

All pair-wise comparisons between the age that each habit was first used were highly significant $(P S<.001)$, except for the comparison of areca nut alone and betel-quid $(P=.9)$. This model also showed that boys were at a significantly higher risk of beginning use before 10 than girls (OR: $2.7295 \% \mathrm{CI}: 1.56,4.73 ; \mathrm{z}=3.54, P<.001$ ).
The effect of sex did not differ between habits $(\mathrm{F}(3,451)=.06$, $P=.98)$.

\section{Frequency of use within current users}

The mean number of uses per week reported by those currently engaging in any areca nut habit is shown in Figure 4. With the exception of pan masala in boys, the frequency of engaging in any of the habits is consistently between around 3 and 5 episodes per week for boys and girls. Pan masala however is used around 10 times a week by boys and it can be seen that boys used all habits more frequently than girls. A multivariate model shows no overall difference in the frequency with which the habits were used per week $(\mathrm{F}(3$, $281)=1.11, P=.3)$. There is, however, a significant interaction between sex and habit $(\mathrm{F}(3,281)=4.45, P=.005)$ indicating that the effect of sex differs between the habits. Analyses of the habits separately shows that boys used pan masala more frequently than girls $(\mathrm{t}=3.18, \mathrm{df}=80, P=.002)$. For areca nut alone $(\mathrm{t}=1.95, \mathrm{df}=$ $217, P=.05)$ and betel-quid $(\mathrm{t}=1.87, \mathrm{df}=139, P=.06)$ the effect of sex on frequency of use is of borderline significance and non-significant in mistee pan $(P=.6)$.

Significant age trends in frequency of use across all habits were also identified in the multivariate model $(\mathrm{t}=2.03, \mathrm{df}=281, P=.04)$. An increase of .29 (95\% CI: .01, .57) uses per week was associated

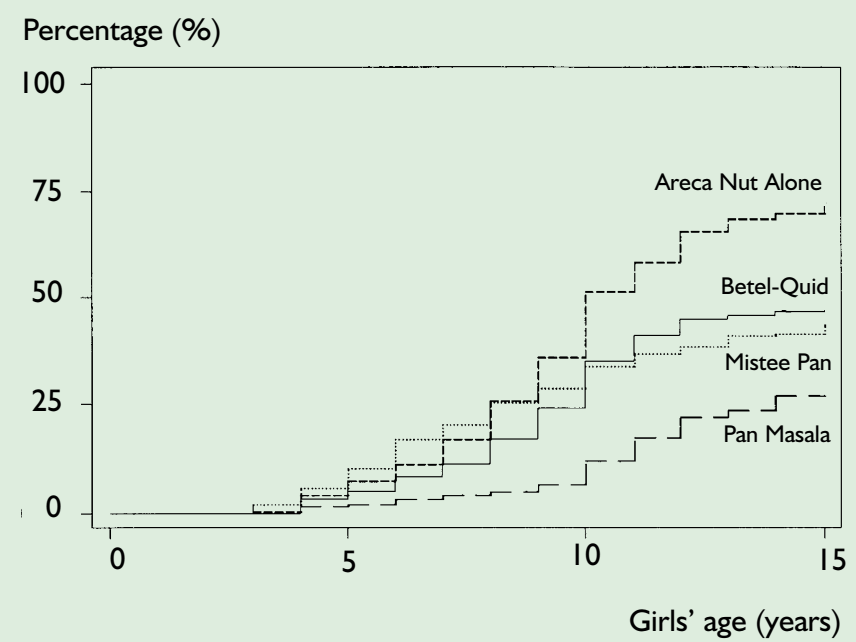

Percentage (\%)



Fig. 2 Retrospective age of first use for each habit in girls and boys 


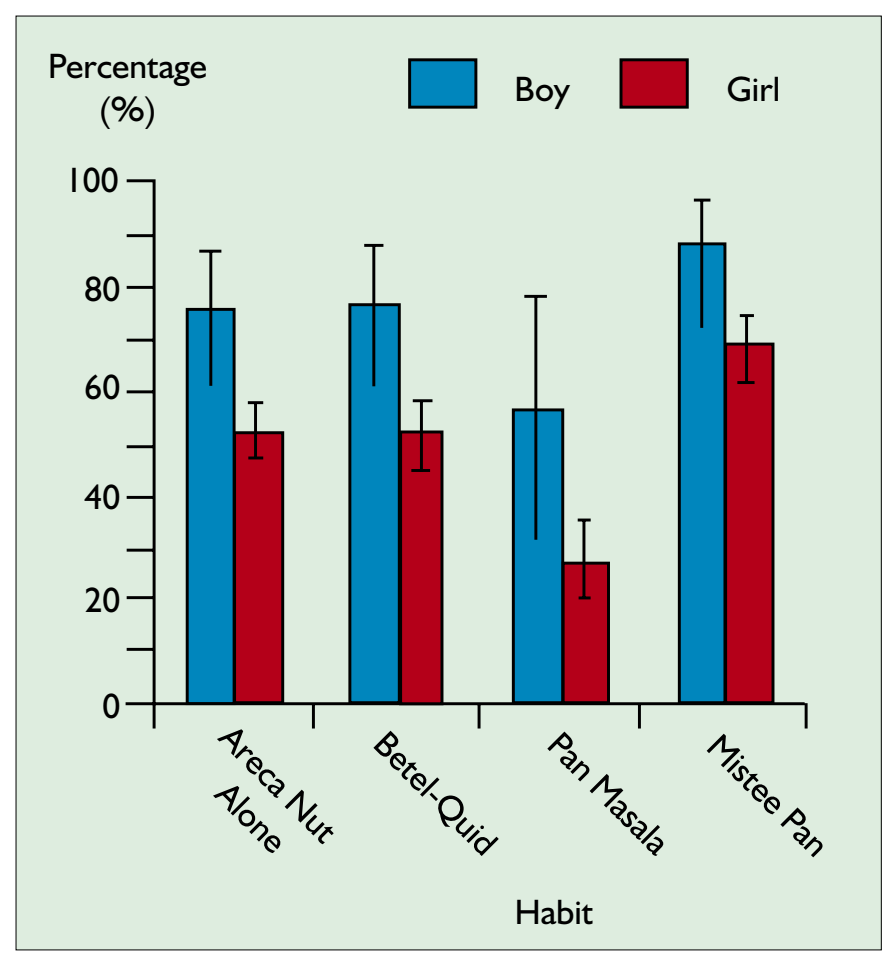

Fig. 3 Percentage (and 95\% confidence intervals) of children who first used each habit before age 10

with a yearly increase in age. Age trends did not differ significantly between habits however $(\mathrm{F}(3,281)=1.69, P=.2)$.

\section{Discussion}

The results from this study indicate that the areca nut habits are highly prevalent within the South Asian population of children examined. There was no evidence to suggest that the habit was extending into other ethnic groups however. A high percentage of South Asian children have used an areca nut habit on at least one occasion with there being a high rate of continuation. Chewing areca nut alone was the most common of the habits currently being used, followed by mistee pan and betel-quid, with a much smaller percentage using pan masala. A high percentage of current users engage in more than one habit. This was especially the case for boys, who were also more likely to begin a habit earlier than girls. The highest period of risk for taking up the habit of chewing areca nut alone, betel-quid and mistee pan for both boys and girls was between the ages of 5 and 12, whilst pan masala was more likely to be taken up after 10 years of age. Additionally the frequency of chewing pan masala was reported to increase with age. Although the lowest proportion of children used pan masala, frequency of use per week by those chewing was by far the highest.

Alarmingly, this study suggests that the highest period of risk for engaging in a betel-quid, areca nut alone or mistee pan habit is between the ages of 5 and 12. This is somewhat earlier than that reported in previous research ${ }^{4}$ for which $11-15$ was cited as the most frequent age range. This difference can be interpreted to suggest that the areca nut habit amongst children with a South Asian ethnic background, but are mainly first or second generation UK born, is being engaged in earlier than amongst previous cohorts born in the South Asian countries. Another explanation is that the data collected in previous studies concerning age of onset, sometimes for periods in excess of 30 years, is largely inaccurate. It has been reported that the reliability and accuracy of retrospective report reduces considerably with increasing lengths of time. ${ }^{16}$

Of additional interest is the finding that areca nut alone was the most commonly used habit amongst children. This contrasts with the situation with an older population in which betel-quid was the most prevalent habit. ${ }^{2,3,13}$ Because of the association between the use of areca nut alone and OSF $F^{6,9,11,12}$ it is possible that by the time the children are entering their teens they already have an increased likelihood of developing OSF. Indeed in India the incidence of OSF amongst children has already been reported. Of 136 patients attending a dental hospital with OSF, 23 per cent were aged between 14 and $19 .{ }^{17}$ In separate studies children as young as $4^{18}$ and $5^{6}$ years old have been diagnosed with OSF. The frequency of using areca nut alone in this study is below that associated with the highest relative risk of developing OSF (between 6-10 chewing episodes per day). ${ }^{11}$ Whilst low however the frequency of using areca nut alone and pan masala did increase with age.

As it is beyond the scope of this paper to establish the extent to which leaf tobacco may be included within the pan masala and betel-quid habits, the findings do indicate a real cause for concern in the event that tobacco is already being added, or becomes added. Previous research has reported a strong relationship between pan masala use and oral cancer. ${ }^{19}$ This relationship is made significantly worse if children engage in a pan masala habit alongside another habit. The findings from this study indicate that pan masala had the highest average frequency of use per week, and furthermore that concurrent use of more than one habit is not uncommon. Additional likelihood of developing oral cancer would also exist if this population continued to engage in an areca nut habit whilst also smoking cigarettes. ${ }^{19}$ Boys would seem to represent a particular risk. They are significantly more likely to engage in an areca nut habit before 10 years of age, have a higher frequency of using areca nut alone, betel-quid and pan masala, and are more likely to engage in cigarette smoking. ${ }^{13}$

That boys rather than girls may potentially be at a higher risk of being exposed to leaf tobacco added to the areca nut habits is contrary to findings carried out within an adult population. ${ }^{6,13}$ In these studies added leaf tobacco was far more prevalent amongst female chewers of betel-quid. A possible explanation for this change is that boys may progress to cigarette smoking with increasing age, therefore no longer adding tobacco to their betel-quid. An additional

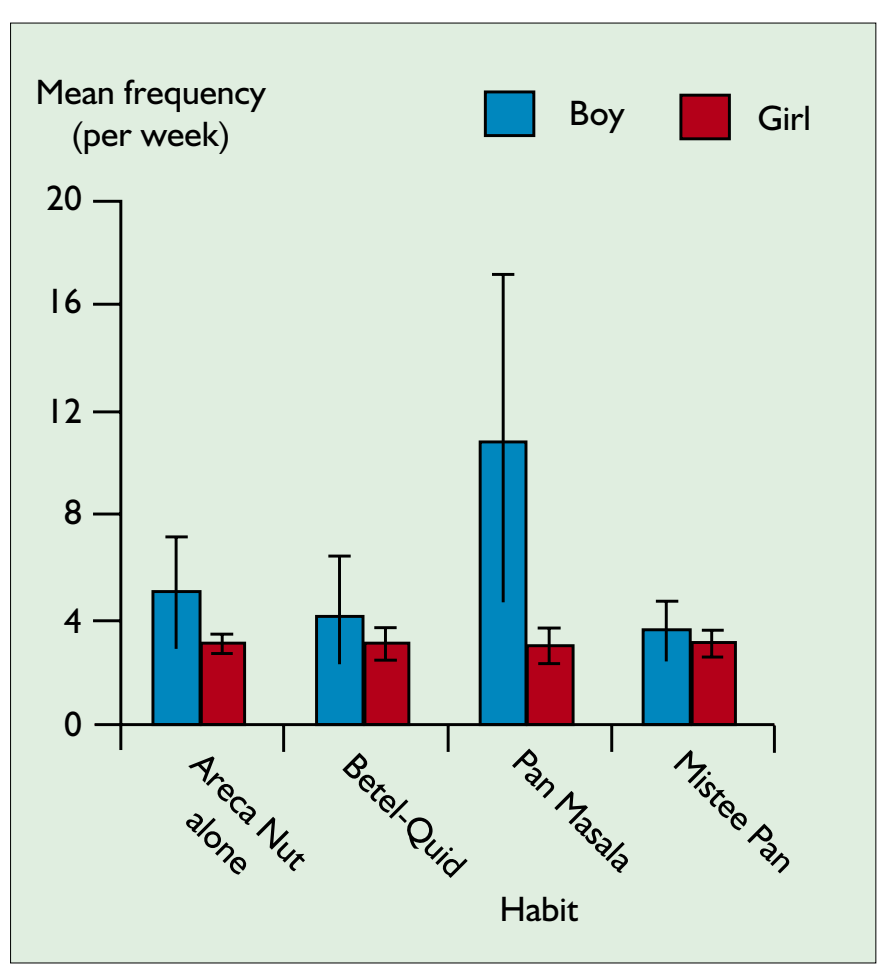

Fig. 4 Mean frequency (and 95\% confidence intervals) of use per week for each habit. 
explanation is that girls engage in any areca nut habit, especially betel-quid, at an even higher age than studied here. Indeed that a habit is engaged in beyond the age of 15 is likely for both boys and girls. Although in far smaller proportions than reported in this study, an areca nut habit has been reported to begin amongst those aged up to 50, with daily frequency of use also increasing with age. ${ }^{4}$ The current cohort of chewers in this study is therefore likely to under represent the size of the future population engaging in an areca nut habit, although the figures are likely to reflect a high percentage of this future population.

The results from this study support the British Dental Association recommendations ${ }^{20}$ which suggest that dentists pay special attention for signs of OSF and potentially malignant lesions amongst South Asian children during routine consultations. Although young, some of these children could have a prolonged history of an areca nut habit, which would be particularly problematic when associated with cigarette smoking or when different habits are engaged in concurrently. However, within the Bangladeshi community in particular, because attendance at dental surgery is low, ${ }^{2,4}$ and the general medical practitioner identified as the person to visit if early signs or symptoms of oral cancer are suspected, ${ }^{3}$ the role of the dentist in early detection may be limited. Other approaches such as the use of health promotion strategies or regulation specifically directed towards reducing the number of children engaging in any areca nut habit may be required.

\section{Limitations}

The first set of limitations is concerned with the terms used to refer to the different areca nut habits. Whilst previously agreed terminology was used in this paper, ${ }^{5}$ commonly used terms differ tremendously. Reliability problems may therefore exist with some of the sample incorrectly reporting their use of a particular habit, a problem even more manifest as the study population consists of children as young as 11 years of age. To minimise the risk of incorrect reporting however, a brief description of each habit was given. Furthermore the fact that marked differences existed across the measures obtained for each habit would suggest reliability problems to have been small. A related limitation is that comment is not possible concerning either the degree to which leaf tobacco is added to the habit or the prevalence of cigarette smoking. Such information would be invaluable, however it was felt that children were unlikely to be aware as to the ingredients added to these habits. Whilst it was originally intended to ask smoking related questions, we were prevented from doing so by the local ethics committee.

The second set of limitations is concerned with how representative the sample of children in the study population are of the wider Tower Hamlets school population. Because of the nature of the study, representative sampling of children could not be completely guaranteed. However that a very high number of children in the participating schools were eligible for free school meals, combined with the high response rate, suggests that the sample largely represents the low socio-economic status experienced by the wider Tower Hamlets school population. Additionally as one of the schools taking part in the study was all girls, the sample consisted of more girls than boys. However analyses for which there were too few boys to be statistically viable were not conducted.

The authors thank all the pupils and staff of Mulberry School for Girls and Oaklands Secondary School, who took part in the study, especially Ms Read and Ms Leahy. Additionally thanks to Chang Park, Girish Purohit and Kumaran Ratnasingham who helped with data collection and Mr Sharif Islam for translating the parent copy of the information and consent form. Finally we are grateful to anonymous reviewer $B$ for many useful comments on this paper.

1 Rudat K. Black and minority ethnic groups in England, health and lifestyle. London: Health Education Authority, 1994.

2 Summers R M, Williams S A, Curzon M E J. The use of tobacco and betel quid ('pan') among Bangladeshi women in West Yorkshire. Community Dent Health 1994; 11: 12-16.

3 Shetty K V, Johnson N W. Knowledge, attitudes and beliefs of adult South Asians living in London regarding risk factors and signs for oral cancer. Community Dent Health 1999; 16: 227-231.

4 Pearson N, Croucher R, Marcenes W, O'Farrell M. Dental service use and the implications for oral cancer screening in a sample of Bangladeshi adult medical care users living in Tower Hamlets, UK. Br Dent J 1999; 186: 517521.

5 Zain R B, Gupta P C, Warnakulasuriya S et al. Oral lesions associated with betel quid and tobacco habits. Oral Cancer 1997; 3: 204-205.

6 Oral Submucous Fibrosis: An expert symposium. Oral Diseases 1997; 3: 276-291.

7 Johnson N W. Orofacial neoplasms: global epidemiology, risk factors and recommendations for research. Int Dent J 1991; 41: 365-375.

8 International Agency for Research on Cancer. IARC monographs on the evaluation of carcinogenic risk of chemicals to humans. Vol 37. Tobacco habits other than smoking: betel-quid and areca nut chewing; and some related nitrosamines. Lyon: IARC, 1985.

9 Murti P R, Bhonsle R B, Gupta P C, Daftary D K, Pindborg J J, Mehta F S. Etiology of oral submucous fibrosis with special reference to the role of areca nut chewing. J Oral Path Med 1995; 24: 145-152.

10 Johnson N W, Warnakulasuriya K. Epidemiology and aetiology of oral cancer in the United Kingdom. Community Dent Health 1993; Suppl 1: 1329.

11 Maher R, Lee A J, Warnakulasuriya K A A S, Lewis J A, Johnson N W. Role of areca nut in the causation of oral submucous fibrosis - a case control study in Pakistan. J Oral Path Med 1994; 23: 65-69.

12 Sinor P N, Gupta P C, Murti P R et al. A case-control study of oral submucous fibrosis with special reference to the etiologic role of areca nut. J Oral Path Med 1990; 19: 94-98.

13 Ahmed S, Rahman A, Hull S. Use of betel quid and cigarettes among Bangladeshi patients in an inner-city practice: prevalence and knowledge effects. Br J Gen Pract 1997; 47: 431-434.

14 Huber P J. The behaviour of maximum likelihood estimates under nonstandard conditions. In Proceedings of the Fifth Berkeley symposium on mathematical statistics and probability 1967. Berkeley: University of California Press I: pp221-223.

15 Kaplan E L, Meier P. Nonparametric observation from incomplete observations. J Am Stat As 1958; 53: 457-481.

16 Pickles A, Neale M, Simonoff E et al. A simple method for censured age-of onset data subject to recall bias - mothers reports of age of puberty in male twins. Behav Genet 1994; 24: 457-468.

17 Babu S, Bhat R V, Kumar P V et al. A comparative clinico-pathological study of oral submucous fibrosis in habitual chewers of pan masala and betel-quid. Clin Toxicol 1985; 34: 317-322.

18 Hayes P A. Oral submucous fibrosis in a 4-year old girl. Oral Surg Oral Med Oral Pathol 1985; 59: 475-478.

19 Bhargava K, Smith L W, Mani N J, Silverman S. Jr, Malaowalla A M, Bilimoria $\mathrm{K}$ F. A follow up study of oral cancer and precancerous lesions in 57,518 industrial workers of Gujarat, India. Ind J Cancer 1975; 12: 124-129.

20 British Dental Association. Occasional Paper Number 6: Opportunistic oral cancer screening. London: British Dental Association, 2000. 